\title{
Le pouvoir des femmes : des Parques aux Matres
}

\section{Véronique Dasen}

\section{(2) OpenEdition}

Journals

Édition électronique

URL : http://journals.openedition.org/edl/146

DOI : $10.4000 /$ edl. 146

ISSN : 2296-5084

\section{Éditeur}

Université de Lausanne

\section{Édition imprimée}

Date de publication : 15 décembre 2011

Pagination : 115-146

ISBN : 978-2-940331-26-0

ISSN : 0014-2026

\section{Référence électronique}

Véronique Dasen, «Le pouvoir des femmes : des Parques aux Matres », Études de lettres [En ligne], 3-4 I 2011, mis en ligne le 15 décembre 2014, consulté le 18 décembre 2020. URL : http:// journals.openedition.org/edl/146 ; DOI : https://doi.org/10.4000/edl.146 


\section{LE POUVOIR DES FEMMES : DES PARQUES AUX MATRES}

Qui possède le droit de vie et de mort sur le nouveau-né? Quand l'enfant reçoit-il une première identité? L'examen du rôle de la sage-femme dans le monde romain remet en question de nombreuses idées reçues. En coupant le cordon, la sage-femme assure le passage du nouveau-né dans le monde des vivants sous le regard de divinités qui confèrent à son geste une valeur rituelle. Bien avant le jour de nomination, une semaine après la naissance, un premier cycle est bouclé quand on procède au premier bain qui intègre l'enfant ici-bas. Loin d'être soumises au contrôle d'un pater familias tout-puissant, ce sont les femmes, humaines et divines, qui président à l'entrée dans la vie.

Dans le monde romain, de nombreuses figures féminines, humaines et surnaturelles, se pressent autour du nouveau-né. Aux yeux des historiens modernes, leur rôle est longtemps apparu secondaire; le sort de l'enfant était jugé entièrement suspendu à un geste du père, suggéré par l'expression tollere ou suscipere liberum, "soulever l'enfant», présenté comme un acte rituel à valeur légale consistant à relever le bébé de terre pour reconnaître sa légitimité. Une relecture attentive des sources antiques, notamment juridiques et médicales, alliée à l'apport de l'iconographie, révèle que ce cérémonial paternel est une construction moderne. Le pouvoir de vie et de mort sur l'enfant nouveau-né revient aux femmes, mortelles et divines, qui président à l'entrée dans la vie. Le discours des images supplée le silence des textes et éclaire la dimension rituelle du rôle de la sage-femme, dont les gestes sont assistés par les divinités du Destin. 


\section{Le pouvoir du père: tollere (suscipere) liberum, levare infantem}

Les historiens modernes ont longtemps affirmé que la puissance paternelle se manifestait à Rome par un rite spécifique: immédiatement après la naissance, l'enfant aurait été déposé à terre, puis relevé par le père qui déclarait par ce geste sa légitimité ${ }^{1}$ :

Le père doit accomplir un geste pour l'accepter dans sa famille. [...] L'enfant est sur le sol, il doit le ramasser, en le prenant par-dessous et l'élever dans ses bras [...]. La naissance physique n'est pas l'essentiel et tant qu'il est à terre l'enfant est inexistant, c'est le geste paternel qui l'appelle à la véritable existence ${ }^{2}$.

A cette reconnaissance paternelle aurait succédé la naissance sociale de l'enfant lors de la fête du dies lustricus, huit jours après la naissance pour une fille, neuf jours pour un garçon, où l'octroi d'un nom parachevait son entrée dans la famille et la communauté.

Th. Köves-Zulauf, puis B. Shaw ont démontré que ce rituel paternel n'existe pas, car l'expression tollere, suscipere ou levare liberum (infantem) n'a qu'une valeur métaphorique ${ }^{3}$. Aucun texte juridique ne mentionne cet acte paternel, également absent des déclarations de naissance d'Egypte romaine ${ }^{4}$. L'acquisition de la puissance paternelle ne dépend pas de l'existence de ce geste. Du point de vue légal, seul compte le fait de naître vivant de l'union de deux citoyens romains, ou iustum matrimonium. Le nouveau-né passe alors automatiquement sous la patria potestas.

Des auteurs modernes affirment aussi que l'accueil des filles à la naissance était différent: aucun cérémonial n’aurait été exécuté pour une fille que le père aurait simplement ordonné d'allaiter ${ }^{5}$. Cette observation

I. Ce topos a suscité une abondante littérature. Parmi les classiques, qui partent du rite comme démontré, voir N. Belmont, «Levana, ou comment “élever” les enfants».

2. J.-P. Néraudau, Etre enfant à Rome, p. 210.

3. Th. Köves-Zulauf, Römische Geburtsriten; M. Corbier, "La petite enfance à Rome», sp. p. 1261-1263; B. D. Shaw, "Raising and Killing Children».

4. Voir p. ex. F. Schulz, "Roman Registers of Birth and Birth Certificates»; id., «Roman Registers of Birth and Birth Certificates. Part II».

5. Y. Thomas, "A Rome, père des citoyens et cité des pères (II ${ }^{\mathrm{e}}$ siècle avant J.-C. - II ${ }^{\mathrm{e}}$ siècle après J.-C.)», p. 198: "S’il s'agissait d'une fille, il ordonnait simplement de la mettre au sein. "Alimenter" (ali iubere) une fille était ainsi une manière de dire qu'on la laisserait vivre, alors que la première nourriture du garçon venait en 
repose sur l'interprétation fautive d'un passage de Suétone relatif à l'empereur Claude qui aurait fait exposer sa fille ${ }^{6}$. Le contexte montre que Suétone dénonce le comportement de Claude qui abandonne un enfant que l'on a déjà commencé à élever, sans indiquer depuis combien de temps, ni sous-entendre de traitement particulier dû à son sexe ${ }^{7}$.

Formulé à la fin du XIX ${ }^{\mathrm{e}}$ siècle, le mythe du cérémonial paternel s'inscrit si bien dans notre représentation de la société patriarcale romaine qu'il a eu la vie dure. Pour justifier l'absence de sa mention dans les textes juridiques, on a préféré faire l'hypothèse d'un "rituel très ancien, précivique " ${ }^{8}$, d'un droit coutumier ancestral, plutôt que de remettre son existence en question.

La confusion des modernes semble provenir d'un amalgame avec les textes médicaux qui décrivent l'acte de poser l'enfant par terre après la naissance. Mais aucun n'ajoute qu'un homme le relève. L'identité de la personne qui accomplit ce geste est pourtant indiquée: il s'agit de la sage-femme, dont les actes ont une dimension rituelle qui est restée inaperçue, éclipsée par le mythe du cérémonial du pater familias.

\section{Le pouvoir de la sage-femme}

A l'époque romaine, l'entrée dans la vie se déroule normalement dans l'espace privé de la domus. Les femmes fortunées peuvent recourir aux services d'une sage-femme, une obstetrix, généralement une esclave ou une affranchie?.

Parmi les textes médicaux antiques, le traité sur les Maladies des femmes de Soranos d'Ephèse (II ${ }^{\mathrm{e}}$ s. apr. J.-C.) livre un témoignage

conséquence d'un geste par quoi le père intégrait son fils dans la suite des pouvoirs hérités et transmis."

6. Suétone, Claude, 27.

7. R. Schilling, «Recension de Léontine Louise Tels-de Jong: Sur quelques divinités romaines de la naissance et de la prophétie», p. 652, relève avec justesse une asymétrie qui passe inaperçue: si le dies Lustricus du neuvième jour pour les garçons est présidé par Nundina, aucune * Octana n'est attestée pour les filles.

8. J. Cels-Saint-Hilaire dans son commentaire à L. Capogrossi Colognesi, «Tollere Liberos", p. 123.

9. M. Eichenhauer, Untersuchungen zur Arbeitswelt der Frau in der römischen Antike, p. 217-245. 
précieux sur le déroulement d'un accouchement. Il met en valeur les compétences de la sage-femme dont il détaille le rôle à chaque étape du processus de la délivrance. Sitôt l'expulsion réussie, c'est elle qui observe s'il s'agit d'un garçon ou d'une fille et l'annonce d'un signe, non précisé, aux personnes présentes ${ }^{10}$. Puis elle accomplit le geste fatidique: elle place l'enfant par terre, avant que le cordon ombilical ne soit coupé, afin d'inspecter sa vigueur et son intégrité physique. Soranos consacre un chapitre entier à cette inspection qui représente une véritable sélection:

La sage-femme, donc, après avoir reçu le nouveau-né, le posera d'abord à terre [...]. Qu'elle se rende compte ensuite si l'enfant vaut ou non la peine qu'on l'élève ${ }^{11}$.

Le mode d'évaluation est complexe: la sage-femme doit tenir compte de l'état de santé de la femme pendant la grossesse, de la durée de la gestation, si possible de neuf mois, voire de sept. Elle se livre ensuite sur l'enfant à un examen systématique de son corps et de sa vigueur: le nouveau-né doit pleurer avec force, ses différents orifices doivent être correctement ouverts, les membres et les articulations souples et mobiles; la sensibilité du nouveau-né est enfin testée par une pression des doigts. Mustio, dans son adaptation latine de Soranos ( $\mathrm{VI}^{\mathrm{e}}$ s. apr. J.-C.), insiste sur le souci de la réactivité de l'enfant qui se traduit par ses cris:

Qu'il crie d'une voix forte lorsqu'il tombe, mais surtout qu'il crie lorsqu'il aura été piqué ou lorsque l'on aura légèrement appuyé sur son corps avec les doigts. Tous ces signes témoignent de sa capacité à ressentir et montrent que l'enfant est né à terme ${ }^{12}$.

Chez Varron, la sage-femme tient droit l'enfant en le relevant afin de le tester:

L'enfant né, il était soulevé de terre par la sage-femme qui le mettait debout pour voir s'il était droit ${ }^{13}$.

IO. Soranos, Maladies des femmes, 2.1.

II. Soranos, Maladies des femmes, 2.5.

I2. Mustio, Gynaecia, 1.76 (trad. Brigitte Maire).

13. Natus si erat vitalis sublatus ab obstetrice, statuebatur in terra, aut auspicaretur rectus esse; Varron, De vita populi romani frgt 81 ap. Nonius Marcellus, De compendiosa doctrina $528 \mathrm{M}$ (éd. citée, p. 848). 
Cet examen détaillé du corps entier, de la tête aux pieds, traduit des préoccupations qui ne se situent pas uniquement sur le plan médical. Il renvoie à la sensibilité particulière du Romain à l'égard des anomalies corporelles qui s'inscrit dans une longue tradition remontant à l'époque républicaine. Les naissances d'enfants physiquement anormaux étaient, jusqu'à l'époque d'Auguste, interprétées comme les signes de ruptures de l'ordre cosmique; dans les cas graves, les nouveau-nés étaient éliminés selon des procédures rituelles spécifiques ${ }^{14}$.

A l'époque de Soranos, on ne lit plus sur le corps du bébé les indices de désordre cosmique, mais toutes sortes de présages sur l'avenir de l'enfant peuvent être déduits de signes de nature diverse qu'il revient à la sage-femme, la première, d'observer, et sans doute aussi d'interpréter.

Différentes sources nous apprennent que la position de l'enfant dans le ventre de sa mère peut annoncer l'avenir. Naître les pieds en avant est contraire à la nature et annonce un destin malheureux: "La loi naturelle veut que l'homme naisse la tête la première, la coutume qu'il soit porté en terre les pieds les premiers", rapporte Pline l'Ancien ${ }^{15}$. Un nom particulier, Agrippa, ut aegre partos, "enfanté difficilement», désignait l'enfant né de cette manière ${ }^{16}$. D'autres singularités sont de bon augure, comme le fait de naître coiffé, c'est-à-dire couvert de la membrane amniotique. Ce prodige aurait annoncé l'accession au trône du futur empereur Diadématus ${ }^{17}$. Les sages-femmes s'emparent de cette membrane "pour la vendre à des avocats superstitieux, car on prétend qu'elle porte chance aux plaideurs", ajoute l'auteur de l'Histoire Auguste ${ }^{18}$. L'observation d'une tache de naissance pouvait aussi augurer d'un destin exceptionnel. Auguste aurait été désigné comme un futur kosmokrator par la présence d’une constellation de taches concentrées sur la poitrine et le ventre, «qui reproduisaient par leur forme, leur disposition et leur

I4. V. Rosenberger, Gezähmte Götter; A. Allély, «Les enfants malformés et considérés comme prodigia à Rome et en Italie sous la République» et «Les enfants malformés et handicapés à Rome sous le principat»; B. Cuny-Le Callet, Rome et ses monstres; V. Dasen, "L'enfant qui ne grandit pas». Sur le cas particulier de l'hermaphrodisme,

L. Brisson, Le sexe incertain.

I5. Pline, Histoire naturelle, 7.45-46 (ut aegre partos).

I6. Pline, Histoire naturelle, 7.46.

17. "Vie de Diaduménien» in Histoire Auguste, 16.4.2.

I8. Ibid., 16.4.2; N. Belmont, Les signes de la naissance, p. 19-126, sp. p. 20. 
nombre, les étoiles de la figure céleste de l'Ourse» ${ }^{19}$. Une précocité anormale, comme parler au jour de sa naissance, est par contre généralement défavorable. Naître avec des dents, selon leur implantation et le sexe de l'enfant, peut annoncer la ruine de toute la collectivité ${ }^{20}$.

Dans le traité de Soranos, l'examen de la sage-femme se termine sobrement par une petite phrase annonçant que "les signes contraires à ceux qui viennent d'être dits révèlent l'inaptitude" ${ }^{21}$. Elle sous-entend une réalité connue par d'autres sources de l'époque impériale; sans justification religieuse, cette fois, les enfants jugés inaptes pouvaient être impunément supprimés ou abandonnés ( ${ }^{\text {er }}$ s. apr. J.-C.) ${ }^{22}$. Soranos passe sous silence les moyens utilisés pour éliminer un bébé indésiré, mais ses recommandations en suggèrent en creux.

En d'autres termes, avant que le pater familias ou tout autre membre de la famille ne donne l'ordre de procéder aux premiers soins, c'est la sage-femme qui détient la première le pouvoir de vie ou de mort sur le nouveau-né. C'est elle qui annonce son sexe et examine sa viabilité, elle qui observe des signes qui peuvent avoir une valeur prophétique, elle enfin qui le relève afin de procéder à la section du cordon. Le souvenir de ce rôle s'est conservé notamment dans les langues italienne et allemande (die Hebamme, la levatrice: "la femme qui lève») ${ }^{23}$.

Si l'enfant est jugé viable, la sage-femme le relève de terre afin de passer à l'étape suivante, la section du cordon ombilical, qui marque son entrée dans la vie humaine. L'opération, qui fait intimement partie de l'identité de la sage-femme ${ }^{24}$, demande des soins particuliers auxquels de nombreuses croyances, souvent à valeur prophétique, sont attachées. Soranos signale ainsi que les sages-femmes préfèrent employer un morceau de poterie brisée ou de verre, un roseau effilé ou une croûte de

19. Suétone, Auguste, 80; V. Dasen, «Empreintes maternelles», sp. p. 50-51.

20. Pline, Histoire naturelle, 7.68-69 (mais c'est un signe de fortune s'il s'agit de la mâchoire supérieure droite, comme dans le cas d'Agrippine, la mère de Néron).

2I. Soranos, Maladies des femmes, 2.5.

22. Sénèque, De la colère, 1.5.2.

23. Cités par N. Belmont ("Levana, ou comment “élever” les enfants", p. 78) qui ne fait pas le lien avec les compétences de la sage-femme antique.

24. Le nom de la sage-femme maia est synonyme d'omphalotomos; Hésychius, "maia", éd. citée, p. 621. 
pain, de crainte que l'usage d'un instrument en métal ne présage la mort violente de l'enfant par magie sympathique ${ }^{25}$.

A aucun moment, les sources ne parlent de déposer l'enfant aux pieds du père. Il n'assiste d'ailleurs en principe jamais à l'accouchement, au mieux, s'il est présent dans la domus, donne-t-il l'ordre de procéder, ou non, aux premiers soins. Mais ses ordres peuvent être donnés à l'avance, s'il est en voyage ${ }^{26}$, ou donnés par la mère, même si le père est présent.

Le père se préoccupe d'ailleurs très peu du nouveau-né ${ }^{27}$. Chez Ovide, Ligdus, le père d'Iphis, déclare à son épouse sur le point d'accoucher qu'il faudra supprimer le nouveau-né s'il s'agit d'une fille:

Une fille naît, à l'insu du père; la mère ordonne de l'élever en la faisant passer pour un garçon; on croit à sa parole et personne ne reçoit la confidence de sa supercherie, sauf la nourrice. Le père s'acquitte de ses vœux et donne à l'enfant le nom du grand-père ${ }^{28}$.

Cette absence usuelle de relation se lit dans la lettre de Sénèque à Marullus, un père orphelin d'un fils en bas âge, submergé par le chagrin:

Ce n'est pas là une souffrance, c'est une pincée au cœur, mais dont tu fais une souffrance. Il est certain que ce sera un progrès philosophique si tu supportes courageusement la perte d'un enfant moins connu jusqu'à présent de son père que de sa nourrice ${ }^{29}$.

25. Soranos, Maladies des femmes, 2.6a. Voir aussi Rufus d'Ephèse ap. Oribasius, Libr. Inc. 12, éd. citée, p. 117-118. Sur l'importance de ce moment au Proche-Orient ancien, voir l'article de C. Frank dans ce volume.

26. Apulée, Métamorphoses, 10.23. Voir la fameuse lettre d'Hilarion à son épouse Alis; S. West, "Whose Baby?» (avec d'autres exemples de pères absents ordonnant d'éliminer une fille).

27. Plutarque, De l'amour de la progéniture, 496B: "Car rien n’est si imparfait, si indigent, si nu, si informe, si souillé que l'homme quand on le voit à sa naissance. Il est presque le seul à qui la nature a même refusé un accès immaculé à la lumière. Tout barbouillé de sang et plein de saleté, il fait plus penser à un assassinat qu'à une naissance, il n'est pas bon à toucher, ni à ramasser, ni à couvrir de baisers, ni à prendre dans les bras, sauf pour qui lui porte naturellement amour.»

28. Ovide, Métamorphoses, 9.675-680 et 705-708.

29. Sénèque, Lettre à Lucilius, 99.14. 
Elle se lit aussi en creux dans l'admiration du comportement inhabituel de Caton l'Ancien qui tenait à assister, raconte Plutarque, à la toilette et à l'emmaillotement de son fils ${ }^{30}$.

Le moment crucial, mais si intime, de la coupe du cordon n'apparaît jamais dans l'iconographie, à une exception près que nous verrons plus loin. La représentation du premier bain semble s'y substituer pour évoquer l'entrée dans la vie humaine. L'importance symbolique du premier bain se situe à plusieurs niveaux. Il agrège l'enfant, séparé du corps de sa mère, au monde des vivants. La sage-femme enlève toutes les traces de la vie utérine sur son corps, elle élimine les mucosités des narines, de la bouche et des yeux, elle provoque l'évacuation du méconium. Elle travaille à transformer la nature du corps de l'enfant en le saupoudrant de sel fin afin de raffermir les grains de sa peau trop tendre ${ }^{31}$. Enfin, l'enfant est frotté de produits qui s'ajoutent au sel - mauve, fenugrec, myrte, huiles parfumées - puis baigné dans une eau où ils se mêlent, intégrant l'enfant à un nouvel univers odorant ${ }^{32}$.

La forte valeur symbolique du premier bain explique la faveur de la scène dans le répertoire traditionnel des naissances tant humaines que divines ${ }^{33}$. On la trouve notamment sur une série de sarcophages de l'époque romaine impériale ( $\mathrm{II}^{\mathrm{e}}$ - début $\mathrm{III}^{\mathrm{e}}$ s. apr. J.-C.) qui représentent les principales étapes de la biographie de jeunes défunts ${ }^{34}$. La scène du bain illustre l'épisode de la naissance avec une densité des présences féminines, composées de la mère, de la sage-femme, des aides, ainsi qu'à l'arrière-plan des Parques qui président aux destinées de l'enfant. Le père n'apparaît que dans la scène suivante, liée à l'éducation ${ }^{35}$. D'ordinaire, l'enfant est encore dans la bassine où le premier bain est donné en

30. Plutarque, Caton l'Ancien, 1.20.4.

3I. Hippocrate, Du régime, 1.19: «Les tanneurs étendent, pressent, peignent, lavent: le traitement des enfants est le même."

32. Soranos, Maladies des femmes, 2.6a.

33. Naissance de Dionysos, voir p. ex. F. Matz, Die dionysischen Sarkophage, sp. pl. 80-91, nos 195-206; J. Huskinson, Roman Children Sarcophagi, p. 30-31.

34. N. B. Kampen, "Biographical Narration and Roman Funerary Art»; S. De Angeli, s.v. "Moirai»; R. Amedick, Vita Privata, p. 60-63; S. Dimas, Untersuchungen zur Themenwahl und Bildgestaltung auf römischen Kindersarkophagen, p. 64-74.

35. Ou à l'allaitement de l'enfant: voir le sarcophage d'Ostie conservé à Paris, Louvre Ma 659 (vers 150 apr. J.-C.); R. Amedick, Vita Privata, cat. no 114, pl. 53, 4; S. Dimas, Untersuchungen zur Themenwahl und Bildgestaltung auf römischen Kindersarkophagen, cat. $\mathrm{n}^{\circ} 386$, pl. 2,4 . 
Fig. 1 - Sarcophage en marbre, de Rome.

Los Angeles County Museum (170-180 apr. J.-C.).

présence de sa mère, identifiée par sa pose alanguie évoquant la fatigue de l'accouchement (fig. 1) ${ }^{36}$. Souvent, la sage-femme a sorti l'enfant nu du bain et le tient debout (fig. 2-6), comme le décrit Varron ${ }^{37}$. Le bébé est toujours figuré plus grand que son âge, plein de vigueur, personnifiant l'enfant qui va survivre. Très actif, il tourne son visage vers sa mère et semble chercher son regard (fig. 1), ou il lui tend les bras (fig. 2; fig. 7).

Il se pourrait que la scène concentre aussi l'étape du dies lustricus, une fête familiale qui se déroule huit ou neuf jours après la naissance, et qui

36. Voir aussi p. ex. le sarcophage de la Via Portuense (vers 100 apr. J.-C.), conservé à Rome, Musée national 125605; R. Amedick, Vita Privata, cat. nº 178, pl. 60, 1.

37. L'enfant s'agrippe à la sage-femme sur le sarcophage conservé à Rome, Musée national 112327; N. B. Kampen, "Biographical Narration and Roman Funerary Art», pl. 10, fig. 18; R. Amedick, Vita Privata, cat. no 179, pl. 63, 1-2. Sur le sarcophage d'Ostie 1170, deux femmes l'enveloppent d'un linge, tandis que la troisième semble tenir un récipient contenant peut-être une huile de massage; N. B. Kampen, "Biographical Narration and Roman Funerary Art», pl. 10, fig. 16; R. Amedick, Vita Privata, cat. $\mathrm{n}^{\mathrm{o}}$ 107, pl. 57, 3. 


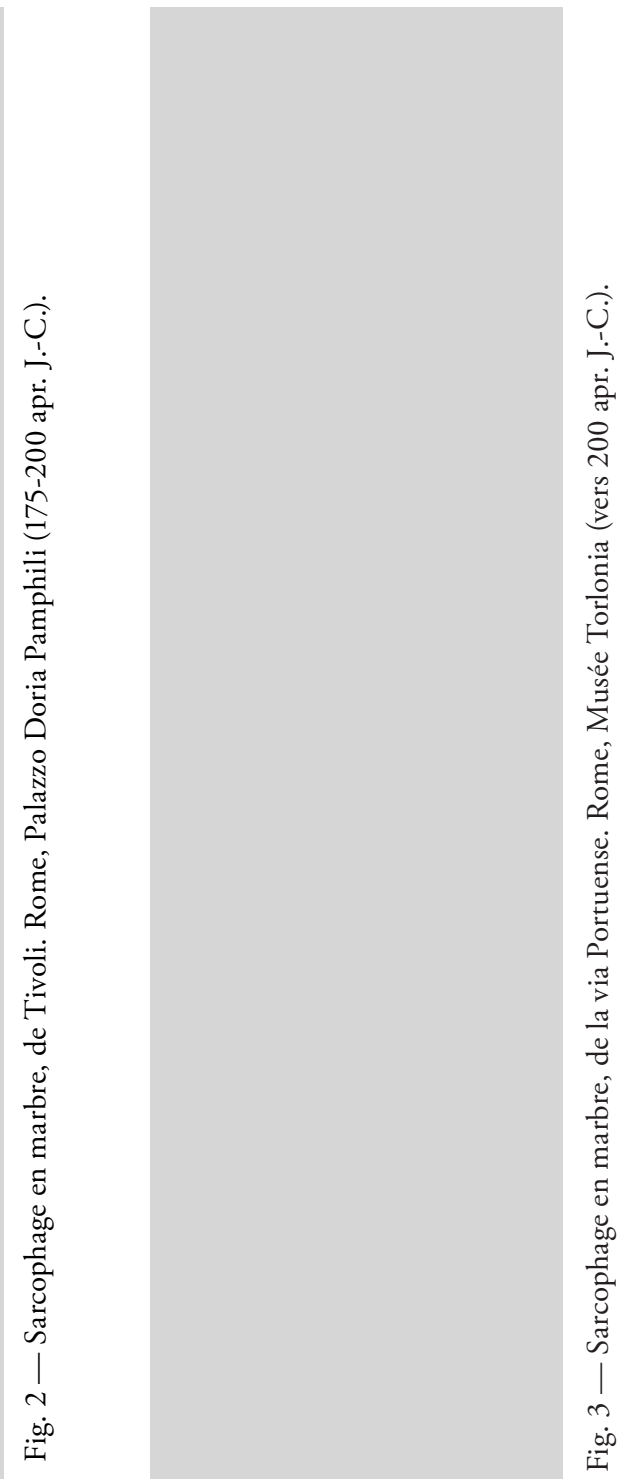


Fig. 4 - Sarcophage en marbre. Florence, Galerie des Offices 82 (vers 150-170 apr. J.-C.).

Fig. 5 - Sarcophage en marbre. Paris, Louvre MA 319 (vers 170-200 apr. J.-C.). 
Fig. 6 - Sarcophage en marbre. Agrigente, Musée national (vers 120-130 apr. J.-C.).

Fig. 7 - Sarcophage en marbre, autrefois à Tivoli. 
Fig. 8 - Autel en marbre. Aquileia, Musée national 364

( $1^{\text {re }}$ moitié du II ${ }^{\mathrm{e}}$ s. apr. J.-C.). 
semble avoir compris des rites de purification ${ }^{38}$. L'image du bain pourrait ainsi résumer les deux moments extrêmes de l'entrée dans la vie, de la section du cordon ombilical aux lustrations du jour de nomination.

Sur les sarcophages, à l'issue du bain, l'enfant est présenté par la sagefemme à sa mère, et non à son père. Ce moment constitue aussi une étape déterminante. La présentation de l'enfant à la mère peut se solder par un rejet. Un autel d'Aquilée dédié à Priape (II ${ }^{\mathrm{e}}$ s. apr. J.-C.) associe le moment du premier bain à celui de l'abandon de l'enfant par sa mère (fig. 8) ${ }^{39}$. Le scholiaste des Argonautiques d'Apollonios de Rhodes raconte qu'Héra, jalouse de la grossesse d'Aphrodite, enceinte de Zeus, toucha le ventre de sa rivale pour éviter que l'enfant à venir ne surpasse en beauté les autres enfants de Zeus. Elle provoqua la difformité du petit Priape (tekeîn amorphon), affligé d'un sexe trop grand pour son âge ${ }^{40}$. Sur le relief d'Aquilée, le bain se déroule en plein air, dans un paysage rocailleux, où la naissance a dû avoir lieu, avec l'assistance de Nymphes. L'une d'elles sort l'enfant de la bassine, posée à l'ombre d'un arbre, et regarde la déesse. Derrière elle, une deuxième femme lève la main pour exprimer son étonnement en détournant son visage de peur ou de dégoût devant l'apparence de l'enfant. A droite, Aphrodite, debout, est négligemment drapée dans un manteau qui dévoile une beauté aussi parfaite que son fils est laid. Sa posture exprime sa décision: elle est en train de s'éloigner et détourne son visage incliné en faisant un geste de refus de la main droite; de sa main gauche, elle tient un pan de vêtement près de son visage, prête à se voiler la face. Aucun regard ne se pose sur l'enfant allongé en position ventrale qui, lui, tourne la tête vers le spectateur pour solliciter son attention.

38. Macrobe, Saturnales, 1.16.36: «Nundina est également une déesse romaine, qui tire son nom du neuvième jour suivant la naissance, dit jour lustral. Quant à ce jour lustral, c'est celui où les nouveau-nés sont purifiés et reçoivent un nom, mais il est le neuvième pour les garçons, le huitième pour les filles.»

39. V. Santa Maria Scrinari, Catalogo delle sculture romane, $\mathrm{n}^{\circ} 554$, fig. 554a-c; W.-R. Megow, s.v. «Priapos», no 168 (fig.).

40. M. Olender, "L'enfant Priape et son phallus», p. 150-155; id., "Priape le mal taillé», p. 375-376. 


\section{Le pouvoir des Parques}

La religion romaine connaît de nombreuses entités surnaturelles qui veillent sur l'ensemble du processus de la procréation, de la conception aux premiers pas de l'enfant. Augustin (IVe s. apr. J.-C.) livre la liste de ces présences invisibles, issue de l'œuvre perdue de Marcus Terentius Varro (I ${ }^{e r}$ s. av. J.-C.) : Diespater conduit les accouchements à terme, Mena veille au flux menstruel (et par là sans doute aussi à la bonne alimentation du foetus), Lucina assiste la délivrance, Opis recueille l'enfant quand il est posé à terre, Vaticanus patronne son premier cri, Levana le moment où il est soulevé de terre par la sage-femme ${ }^{41}$. Tertullien (fin $\mathrm{II}$ s. apr. J.-C.) complète l'énumération avec des variantes: Consevius, Fluvionia, Vitumnus, Sentinus président à la vie intra-utérine, Diespater amène l'enfant à la lumière du jour avec Candéliféra ${ }^{42}$.

L'accouchement, ainsi que la position de l'enfant dans le ventre maternel, sont patronnés par des divinités dont les compétences prophétiques relaient sur le plan surnaturel les observations de la sage-femme. La plus célèbre est Carmentis ou Carmenta qui détient une parole inspirée, comme le suggère son nom, dérivé de carmen, qui désigne le chant ou une formule incantatoire ou magique. Varron, chez Augustin, explique que les Carmentes prédisent l'avenir des enfants à l'accouchement, nascentibus ${ }^{43}$. Dans ses Questions romaines, Plutarque précise que Carmenta est l'équivalent d'une Moire, qui a des compétences similaires en Grèce ${ }^{44}$. Comme les Moires, Carmenta peut se transformer en figures plurielles, les Carmentes, sous la forme d'une paire ou d'une triade avec des noms différents. Varron décrit leurs compétences obstétricales:

Lorsque, d'une manière contre nature, ils se retournent les pieds vers le bas, que, les bras généralement écartés, ils résistent et qu'alors les femmes ont un accouchement plus douloureux, pour éloigner ce danger par des pratiques religieuses on a élevé à Rome des autels aux deux Carmenta dont l'une a été surnommée Postuerta (tournée vers l'arrière)

4I. Varron, Antiquitates rerum humanarum et divinarum, ap. Augustin, Cité de dieu, 4.11.2-3.

42. Tertullien, Contre les nations, 2.11.1-6.

43. Augustin, Cité de Dieu, 4.11: Quae fata nascentibus canunt et vocantur Carmentes. Ovide, Fastes, 1.633 sq. : Posverta prédit l'avenir de l'enfant.

44. Questions romaines, 56 (278C): «Carmenta est une Moire.»Sur les compétences des Moires, voir dans ce volume l'article de V. Pirenne-Delforge et G. Pironti. 
et l'autre Prorsa (tournée vers l'avant), des conditions et du nom de l'accouchement normal et du mauvais ${ }^{45}$.

A côté des Carmentes, d'autres noms circulent, au singulier ou au pluriel. Les plus familiers sont Parcae et Fata, des divinités intimement liées à la valeur prophétique du moment de la naissance. Selon Varron, Fata viendrait de fare, parler "parce que les Parques (Parcae) déterminent alors en parlant (fando) le temps de vie des enfants » ${ }^{46}$, d'où le nom fatum (destinée) et fatidicus (qui prédit l'avenir). Ailleurs, il précise que Parca est l'une des Tria Fata qui président la naissance avec Nona et Decima:

Varron dit que les anciens Romains [...] ont donné aux trois Destinées (tribus Fatis) des noms tirés de parere, enfanter, de nonus et decimus, neuvième et dixième mois. Parca, la Parque, vient de partus, enfantement, après changement d'une seule lettre; Nona, et Decima, du moment favorable pour l'accouchement ${ }^{47}$.

Comment se traduisent visuellement les compétences de ces divinités? La fluidité de leurs identités ne permet pas toujours de les reconnaître aisément. La série la plus importante de documents se rapporte aux Parques, au nombre de trois, sur le modèle des Moires grecques. On les trouve principalement sur des monuments funéraires de l'époque romaine impériale où leur présence fait écho au motif littéraire de la Moire ou Parque inflexible, bien attesté dans les inscriptions funéraires latines. Leur présence est d'ordinaire le signe d'une destinée tragique, achevée par une mort prématurée sur les sarcophages biographiques d'enfants, ou annoncée sur les sarcophages mythologiques (Méléagre, Icare, Phaéton, Hippolite...). Les divinités sont identifiées par des attributs en partie hérités des Moires grecques, comme la quenouille et le fuseau, en partie nouveaux, comme le volumen ou livre du Destin, peut-

45. Aulu-Gelle, Nuits attiques, 16.16.2-4. Voir aussi Macrobe, Saturnalia, 1.7.20 (Antevorta et Postvorta). Antevorta, Pro(r)sa et Postvorta déterminent la position de l'enfant au moment de l'expulsion, par la tête ou les pieds. Si l'enfant se présente de travers, on invoque la déesse Prorsa Carmentis, qui doit le pousser en avant. N. Belmont, Les signes de la naissance, p. 161-180 ; J.-J. Aubert, "La procréation divinement assistée dans l'Antiquité gréco-romaine».

46. Varron, De la langue latine, 6.52

47. Aulu-Gelle, Nuits attiques, 3.16.9-10. 
être hérité du monde étrusque ${ }^{48}$, la balance, empruntée à l'iconographie d'Aequitas ou de Némésis, et la roue, empruntée à Némésis (fig. 2).

A la différence des textes, qui exploitent l'image du fil rompu ou coupé pour traduire l'achèvement de la vie ${ }^{49}$, l'iconographie développe d'autres métaphores. Sur les sarcophages d'enfants, le fuseau est d'ordinaire absent ${ }^{50}$; il est remplacé par le globe qui se rapporte à la pratique de l'astrologie, en vogue à l'époque impériale. A l'arrière-plan de la scène du premier bain, les déesses sont réunies. L'une d'elles pointe sa baguette sur la sphère céleste pour indiquer le signe zodiacal de la naissance, tandis qu'une autre tient le livre où son horoscope ou son destin sera inscrit $^{51}$ (fig. 1, 4-6). La scène pourrait aussi se rapporter au jour du dies lustricus ou jour de nomination; selon Tertullien, on inscrivait alors les fata scribunda, les présages ou paroles omineuses prononcées par les personnes présentes ou les déesses présidant au destin ${ }^{52}$.

La présence des Parques est rare en dehors du contexte funéraire. Elles président de manière solennelle à la naissance d'Achille sur la mosaïque de la maison de Thésée à Néa Paphos, Chypre (fin IV - début $\mathrm{V}^{\mathrm{e}} \mathrm{s}$. apr. J.-C.) (fig. 9). Clotho, Lachésis et Atropos se tiennent debout, tête voilée, derrière Thétis et Pélée qui s'apprêtent à assister au premier bain du nouveau-né, assis sur les genoux de sa nourrice (anatrophè), le dos bien droit, les bras tendus. Chacune présente des objets qui se rapportent à la détermination du destin du héros, avec un redoublement sur le thème de l'écriture. Le jeu de leurs regards, qui embrassent l'ensemble de l'espace, souligne leurs pouvoirs surnaturels: Clotho file le cours de l'existence avec la quenouille et le fuseau en regardant l'enfant; Lachésis, de face, tient un stylet pour inscrire le destin sur la tablette, tandis qu'Atropos

48. F. Gury, «La Forge du Destin», sp. p. 458-459.

49. S. Ballestra-Puech, Les Parques, p. 83-88. Voir aussi le thème de l'épuisement de la masse de laine dans S. Ballestra-Puech, Les Parques, p. 79-83.

50. Fait exception la triade avec globe, volumen et fuseau sur le sarcophage du Vatican, Museo Chiaramonti 1632; S. Dimas, Untersuchungen zur Themenwahl und Bildgestaltung auf römischen Kindersarkophagen, pl. 5, 3, cat. 396; S. De Angeli, "Problemi di iconografia romana», p. 118, fig. 4.

5I. Sur les questions de prédestination astrologique à l'époque romaine impériale, V. Dasen, "Naître jumeaux: un destin ou deux?».

52. Tertullien, De anima, 39: dum ultima die Fata Scribunda advocantur. Sur l'identité débattue des fata scribunda, voir la discussion de L. L. Tels-de Jong, Sur quelques divinités romaines de la naissance et de la prophétie, p. 105-129. 
Fig. 9 - Mosaïque. Villa de Thésée, Néa Paphos, Chypre (fin $\mathrm{IV}^{\mathrm{e}}$ - début $\mathrm{V}^{\mathrm{e}}$ s. apr. J.-C.).

déroule un volumen en tournant son regard vers d'autres horizons, à l'extérieur de la scène ${ }^{53}$.

La figure des Parques ne se réduit pas à leur face sombre et inquiétante, annonciatrice d'un destin abrégé. En Gaule romaine, les déesses possèdent une dimension bienveillante ${ }^{54}$, visible sur une série de reliefs votifs qui devaient appartenir à des chapelles domestiques. Ces

53. Cf. Iliade, 20.125-128: "plus tard il devra subir ce que la Destinée a filé pour lui à sa naissance, lorsque sa mère l'enfanta. » Sur la présence possible d'une Parque dans les scènes de nativité, I. Jevtic, «L'inscription dans la vie».

54. Cette face est aussi connue au Moyen Age où les Parques se confondent avec les trois Marie; S. Ballestra-Puech, Les Parques, p. 330-331. Cette ambivalence se traduirait-elle par la distinction entre "bonnes» et "mauvaises" fées dans la tradition ultérieure? 
Fig. 10 - Groupe en calcaire, du sanctuaire des Bolards. Dijon, Musée archéologique (Haut-Empire).

monuments représentent une triade de femmes, nommées Matres, qui veillent sur l'enfant. Un examen de leurs attributs montre qu'il s'agit de figures similaires aux Parques. Le groupe en haut-relief de Vertault (Côte d'Or) représente trois femmes assises sur un siège à haut dossier ${ }^{55}$. Elles portent sur leurs genoux, l'une le nourrisson, la deuxième le linge, la dernière l'éponge et la cuvette. Comme $S$. Deyts l'avait bien relevé ${ }^{56}$, il ne s'agit probablement pas d'une simple évocation des soins donnés au tout-petit, un deuxième niveau de lecture est implicite. Les trois femmes ont un sein dénudé qui signale leurs qualités nourricières, le diadème posé sur leur chevelure indique qu'il s'agit de déesses; le linge pourrait représenter un parchemin déroulé, la cuvette une patère à libation. Ce deuxième niveau de lecture est confirmé par la présence d'une balance dans deux autres triades féminines. Sur le relief des Bolards (fig. 10) ${ }^{57}$

55. Châtillon-sur-Seine, Musée du Châtillonais 88.171.1; S. Deyts, Images des dieux de la Gaule, p. 65 (fig.); G. Bauchhenss, s.v. "Matres, Matronae», nº 38 (fig.).

56. S. Deyts, Images des dieux de la Gaule, p. 64-66 (fig.).

57. G. Bauchhenss, s.v. «Matres, Matronae», no 43. 
Fig. 11 - Gemme en verre. Londres, British Museum (Irer s. av. J.-C.).

Fig. 12 - Gemme en verre. Genève, Musée d'art et d'histoire, MF 1947 (I ${ }^{\text {er }}$ s. av. J.-C.). 
ainsi que sur le relief de Saint-Boil ${ }^{58}$, une des déesses tient une balance, tandis qu'à la balance et au volumen s'ajoute le fuseau sur l'autel conservé à Darmstadt ${ }^{59}$. Des inscriptions confirment les affinités de ces figures avec les Parques ou les Moires. Sur un autel conservé à Avignon, les trois femmes, sans attribut, sont appelées "Fata» ${ }^{60}$, tandis que sur un relief de Bucarest l'inscription les nomme «Moires» ${ }^{61}$.

Une série de gemmes et pâtes de verre de l'époque augustéenne ( $\mathrm{I}^{\mathrm{er}}$ s. av. J.-C. - $\mathrm{I}^{\text {er }}$ s. apr. J.-C.) livrent un autre ensemble d'images non funéraires qui proposent une nouvelle lecture de la fonction symbolique de la fileuse dans les scènes de naissance ${ }^{62}$. Sur l'intaille en verre du British Museum, les attributs des divinités sont conformes à la tradition iconographique (volumen, fuseau, balance) (fig. 11). S'ajoutent la présence d'une torche ainsi que celle d'un petit enfant nu, allongé par terre. Le motif se retrouve sur d'autres gemmes, en pierre ou en verre, avec des variantes (fig. 12). Les Parques sont debout, sans attribut, mais toujours avec à leurs pieds une torche et un bébé nu, assis ou allongé.

La torche et l'enfant situent la scène dans le contexte de la naissance. La torche est l'attribut des divinités qui président aux mariages et aux naissances, telles Candéliféra, "parce que les accouchements ont lieu à la lumière d'une chandelle» ${ }^{63}$, et Iuno Lucina, celle qui "amène l'enfant à la lumière ${ }^{64}$. La torche renvoie aussi aux rites de purification qui suivent une naissance.

L'enfant allongé est le nouveau-né sur lequel se penchent les Parques pour fixer son destin au moment de son premier cri, quand la sagefemme le pose par terre et procède à l'examen de sa viabilité. Sur quelques gemmes (fig. 12), l'enfant se redresse et tend les bras vers les femmes qui l'observent. La scène éternise le moment présidé par Levana, lorsque la sage-femme s'apprête à relever l'enfant (statuere infantem) avant de procéder aux premiers soins.

58. S. Deyts, Images des dieux de la Gaule, p. 64 (fig.); G. Bauchhenss, s.v. «Matres, Matronae", $n^{\circ} 37$.

59. G. Bauchhenss, s.v. «Matres, Matronae», no 44.

6o. G. Bauchhenss, s.v. «Matres, Matronae», nº 59.

6I. S. De Angeli, s.v. "Moirai», $n^{\circ} 3$.

62. C. Weiss, "Deae fata nascentibus canunt"; V. Dasen, s.v. "Moirai».

63. Tertullien, Contre les nations, 2.11.1-6.

64. Tertullien, De anima, 37. Voir aussi Ovide, Fastes, 3, sur le culte de Iuno Lucina. 
Fig. 13 - Icône du monastère de Sainte-Catherine du Sinaï (vers 1200). 
L'intaille en verre du British Museum (fig. 11) offre une variante avec des détails supplémentaires. Elle livre la dimension rituelle d'un geste décisif qui n'est d'ordinaire jamais représenté: la section du cordon ombilical. L'image opère par métaphores. La fileuse, de face, occupe la place centrale à côté de l'enfant qui gît au sol, inerte, le lien à sa vie intra-utérine coupé, pas encore réellement vivant. La Parque ne file pas n'importe quel type de fil: dans la littérature latine, il s'agit toujours de "laine moelleuse " ${ }^{65}$. Or Soranos, comme Mustio, insiste beaucoup sur l'emploi de laine, et non de lin, pour ligaturer le cordon coupé:

On ligaturera le bord de la coupure avec par exemple un flocon de laine tordu, un fil de lice, un brin de laine ou tout autre moyen semblable. Le fil de lin, entamant les chairs délicates, cause des douleurs peu supportables ${ }^{66}$.

C'est aussi un tampon ou une houppe de laine que l'on applique ensuite sur l'ombilic ${ }^{67}$. La Parque opère à nouveau comme un double de la sagefemme: la laine qu'elle file est celle qui va ligaturer le cordon de l'enfant posé à terre. La sage-femme attache ainsi symboliquement au corps de l'enfant le fil de la vie que la Parque commence à filer ${ }^{68}$.

Les auteurs médicaux insistent beaucoup sur les dangers de cet acte où la vie et la mort se côtoient. Soranos, comme Mustio, conseille de sectionner le cordon à au moins quatre doigts de distance du corps de l'enfant et de bien le ligaturer pour éviter les hémorragies ${ }^{69}$. Des anecdotes font allusion à l'action malveillante de sages-femmes. On raconte ainsi que le fils d'Hélène et de l'empereur Julien fut éliminé à la naissance par

65. P. ex. Catulle, Poésies, 64.307-319; S. Ballestra-Puech, Les Parques, ch. 2.

66. Soranos, Maladies des femmes, 2.6a. Mustio, Gynaecia, 1.78 (trad. Brigitte Maire) : "Il ne faut pas accepter le rite habituel des Anciens qui le coupaient à l'aide d'un fil de lin ou avec du verre ou alors avec un roseau tranchant ou une croûte de pain. Ensuite, quand on l'aura coupé, on séchera le sang coagulé qui sera resté à l'intérieur et on l'attachera avec de la laine que l'on enroulera tout autour".

67. Soranos, Maladies des femmes, 2.6a.

68. Cf. le bandeau rouge figurant le fil de la vie qui sert d'attribut aux Sept Hathors qui prédisent le destin du nouveau-né; voir dans ce volume l'article de C. Spieser.

69. Certaines sages-femmes cautérisent même le cordon pour éviter tout risque, ce que Soranos (Maladies des femmes, 2.6) désapprouve en raison des douleurs occasionnées. 
une sage-femme corrompue qui aurait coupé délibérément le cordon plus qu'il ne convenait pour supprimer un héritier encombrant ${ }^{70}$.

Ces gemmes portées en bague ou pendentif ont peut-être représenté un cadeau pour une heureuse naissance. Leur thématique révèle, une fois de plus, l'originalité du répertoire des pierres gravées où l'artiste peut librement traiter de sujets intimes attestés sur aucun autre support, comme la protection de la vie utérine et de l'accouchement ${ }^{71}$.

Le parallèle le plus proche du symbolisme de l'intaille du British Museum se trouve dans quelques scènes de l'Annonciation de l'époque médio-byzantine représentant la Vierge filant la laine qui servira à tisser le voile du Temple. Sur une icône du monastère de Sainte-Catherine du Sinaï (début du XIII e siècle) (fig. 13), la Vierge tient dans la main gauche l'écheveau et touche la mandorle où se tient le Christ Enfant nu, peint en grisaille sur sa poitrine, tandis que sa main droite dirige le fuseau vers une sorte de coquille posée sur son giron. L'artiste semble avoir cherché à traduire la préoccupation théologique de l'époque sur la nature humaine du Christ, Logos Incarné ${ }^{72}$. De nombreux textes comparent le tissage du Voile à celui de la chair mortelle du Christ dans le secret du corps virginal de la Théotokos ${ }^{73}$. L'image du fil de laine pourpre pourrait évoquer le cordon ombilical qui relie le Christ à la matrice de la Vierge, symbolisée par la coquille.

\section{Conclusion}

Le discours des images vient pallier le silence des textes et inscrit dans une perspective nouvelle le moment de la naissance dans le monde romain. Si le «cérémonial paternel» n’a jamais existé, l'activité de la sagefemme va bien au-delà du rôle d'une simple technicienne: les premiers gestes de l'entrée dans la vie sont accomplis sous le regard de divinités qui leur accordent une valeur rituelle. Postverta et Prorsa accompagnent le travail de version de l'enfant quand l'accouchement est difficile. Les

70. Ammien Marcellin 16.10.19.

7I. V. Dasen, «Représenter l'invisible».

72. M. Evangelatou, «The Purple Thread of the Flesh». Sur le Logos Incarné dans les scènes de Visitation, voir E. Yota, «L'embryon dans l'art byzantin: une image insolite».

73. Sur la gestation du Christ comparée au tissage, voir N. P. Constas, "Weaving the Body of God». 
Carmentes, Fata et Parcae président à l'observation des signes augurant l'avenir, heureux ou malheureux, en bonne ou mauvaise santé, du nouveau-né. Le décor des intailles révèle une compétence des Parques qu'aucun texte n'explicite. Si Levana accompagne le moment où la sagefemme relève l'enfant de terre, la section du cordon est présidée par les Parques qui filent la laine qui va servir à le ligaturer, reliant l'enfant par son cordon à une vie nouvelle.

Le rôle de "passeuse» de la sage-femme, en contact avec la vie et la mort, lui confère un statut particulier dans l'imaginaire collectif que traduit sa promiscuité avec ses doubles divins. Une inscription d'Izmir du $\mathrm{II}^{\mathrm{e}}$ ou I ${ }^{\mathrm{er}}$ siècle av. J.-C. le résume en évoquant le drame d'une mort en couches où l'actrice surnaturelle et l'actrice humaine se relaient dans la même phrase:

Le même jour, la sage-femme et la Moire s'emparèrent de la jeune fille (korè), elles lui offrirent un heureux enfantement et la mort ${ }^{74}$.

Véronique DASEN

Université de Fribourg

74. Plaque en marbre, Musée d'Izmir 737; E. Samama, Les médecins dans le monde grec, p. 8, n. 4 . 


\section{BIBLIOGRAPHIE}

\section{Sources}

Ammien Marcellin, Histoires, T. I (livres XIV-XVI), texte établi et traduit par E. Galletier avec la collaboration de J. Fontaine, Paris, Les Belles Lettres, 1968.

Apulée, Les métamorphoses, T. III (livres VII-XI), texte établi par D. S. Robertson et traduit par P. Valette, Paris, Les Belles Lettres, 1965.

Augustin, Cité de Dieu, T. I (livres I-V), texte traduit par P. de Labriolle, Paris, Garnier, 1957.

Aulu-Gelle, Les nuits attiques, T. I (livres I-IV), T. IV (livres XVI-XX), texte établi et traduit par R. Marache, Paris, Les Belles Lettres, 1967 et 1978.

Catulle, Poésies, texte établi et traduit par G. Lafaye, Paris, Les Belles Lettres, 1992.

Hesychius, Hesychii Alexandrini Lexicon, ed. K. Latte, Copenhagen, T. 1 (Alpha-Delta), 1953, T. 2 (Epsilon-Omikron), 1966.

Hippocrate, Du régime, texte établi et traduit par R. Joly, Paris, Les Belles Lettres, 1967.

Histoire Auguste, traduction et commentaire d'A. Chastagnol, Robert Laffont, collection Bouquins, 1994.

Macrobe, Saturnales, livres I-III, introduction, traduction et notes par

Ch. Guittard, Paris, Les Belles Lettres (La Roue à livres), 1997.

Mustio, Sorani Gynaeciorum uetus translatio Latina, éd. V. Rose, Leipzig, Teubner, 1882.

Nonius Marcellus, De compendiosa doctrina libros $X X$, éd. W. M. Lindsay, Leipzig, Teubner, 1903.

Pline l'Ancien, Histoire naturelle, livre VII, texte établi, traduit et commenté par R. Schilling, Paris, Les Belles Lettres, 1977. 
Plutarque, Vies, T. V, Caton l'Ancien, texte établi et trad. par R. Flacelière et E. Chambry, Paris, Les Belles Lettres, 1969.

—, Euvres morales, T. IV, traités 17 à 19, Conduites méritoires de femmes, Etiologies romaines - Etiologies grecques, Parallèles mineurs, texte établi et traduit par J. Boulogne, Paris, Les Belles Lettres, 2002.

-, Euvres morales, T. VII, 1ère partie, traités 27-36, De l'amour de la progéniture, texte établi et traduit par J. Dumortier avec la collaboration de J. Defradas Paris, Les Belles Lettres, 2003.

Ovide, Les fastes, T. I (Livres I-III), T. II (Livres IV-VI), texte établi, traduit et commenté par R. Schilling, Paris, Les Belles Lettres, 1992-1993.

-, Les métamorphoses, T. II (livres VI-X), texte établi et traduit par G. Lafaye, édition revue et corrigée par H. Le Bonniec, Paris, Les Belles Lettres, 1995.

Rufus D'Ephèse, Euvres de Rufus d'Ephèse, trad. Ch. Daremberg et E. Ruelle, Paris, Imprimerie nationale, 1879.

Sénèque, Dialogues, T. I, De la colère, texte établi et traduit par A. Bourgery, Paris, Les Belles Lettres, 1971.

—, Lettres à Lucilius, traduction H. Noblot, révisée par P. Veyne, Paris, Les Belles Lettres, 1993.

Soranos D'Ephèse, Maladies des femmes, T. II (livre II), texte établi, traduit et commenté par P. Burgière, D. Gourevitch, Y. Malinas, Paris, Les Belles Lettres, 1990.

SuÉtone, Vies des douze Césars, T. 1, César, Auguste, texte établi et traduit par H. Ailloud, Paris, Les Belles Lettres, 2008.

—, Vies des douze Césars, T. II, Tibère, Caligula, Claude, Néron, texte établi et traduit par H. Ailloud, Paris, Les Belles Lettres, 1989.

Tertullien, Contre les nations, Le premier livre "Ad nationes» de Tertullien, introd., texte, trad. et comment. A. Schneider, Rome, Institut suisse de Rome, 1968.

—, De anima, Über die Seele, eingel., übers. und erl. von J. H. Waszink, Zürich, München, Artemis Verlag, 1980.

Varron, De la langue latine, T. II (livre VI), texte établi, traduit et commenté par P. Flobert, Paris, Les Belles Lettres, 1985. 


\section{Travaux}

AlléLy, Annie, "Les enfants malformés et considérés comme prodigia à Rome et en Italie sous la République", Revue des études anciennes, 105 (2003), p. 127-156.

—, "Les enfants malformés et handicapés à Rome sous le principat", Revue des études anciennes, 106 (2004), p. 73-101.

Amedick, Rita, Vita Privata. Die Sarkophage mit Darstellungen aus dem Menschenleben, Berlin, Gebr. Mann, 1991 (Die antiken Sarkophagreliefs, Bd I, Teil 4).

Aubert, Jean-Jacques, "La procréation divinement assistée dans l'Antiquité gréco-romaine", in Naissance et petite enfance dans l'Antiquité. Actes du colloque de Fribourg, 28 novembre-1 ${ }^{\mathrm{er}}$ décembre 2001, éd. par V. Dasen, Fribourg/Göttingen, Academic Press, Vandenhoeck \& Ruprecht, 2004, p. 187-198 (OBO 203).

Ballestra-Puech, Sylvie, Les Parques. Essai sur les figures féminines du destin dans la littérature occidentale, Toulouse, Editions Universitaires du Sud, 1999.

Bauchinenss, Gerhard, «Matres, Matronae», LIMC VIII, Supplementum (1997), p. 808-816.

Belmont, Nicole, Les signes de la naissance. Etude des représentations symboliques associées aux naissances singulières, Paris, Gérard Monfort, 1971.

—, "Levana, ou comment "élever" les enfants", Annales. Histoire, Sciences Sociales, 28 (1973), p. 77-89.

BrIssOn, Luc, Le sexe incertain: androgynie et hermaphrodisme dans l'Antiquité gréco-romaine, Paris, Les Belles Lettres, 1997.

Capogrossi Colognesi, Luigi, "Tollere Liberos", Mélanges de l'Ecole française de Rome. Antiquité, 102 (1990), p. 106-127.

Constas, Nicholas P., "Weaving the Body of God: Proclus of Constantinople, the Theotokos, and the Loom of the Flesh", Journal of Early Christian Studies, 3 (1995), p. 169-194.

Corbier, Mireille, «La petite enfance à Rome: Lois, normes, pratiques individuelles et collectives", Annales. Histoire, Sciences Sociales, 54/6 (1999), p. 1257-1290.

Cuny-Le Callet, Blandine, Rome et ses monstres, Paris, Jérôme Millon, 2005. 
Dasen, Véronique, "L'enfant qui ne grandit pas", Medicina nei secoli. Storia dell'handicap infantile in Italia, 18 (2006), p. 431-457.

—, «Représenter l'invisible: la vie utérine sur les gemmes magiques», in L'embryon humain à travers l'histoire. Images, savoirs et rites, éd. par V. Dasen, Gollion, Infolio, 2007, p. 41-64.

—, "Naître jumeaux: un destin ou deux?", in L'embryon: formation et animation. Antiquité grecque et latine, traditions hébrä̈que, chrétienne et islamique, éd. par L. Brisson, M.-H. Congourdeau, J.-L. Solère, Paris, Vrin, 2008, p. 109-122.

—, «Empreintes maternelles», in La madre/The mother, Firenze, Sismel, 2009, p. 35-54 (Micrologus. Nature, Science and Society, 17).

—, «Moirai», LIMC, Supplementum (2009), p. 338-339.

De Angeli, Stefano, "Problemi di iconografia romana: dalle Moire alle Parche", Mélanges de l'Ecole française de Rome. Antiquité, 103 (1991), p. 105-128.

—, "Moirai ", LIMC VI (1992) p. 636-648.

Deyts, Simone, Images des dieux de la Gaule, Paris, Errance, 1992.

Dimas, Stephanie, Untersuchungen zur Themenwahl und Bildgestaltung auf römischen Kindersarkophagen, Münster, Scriptorium, 1998.

Eichenauer, Monika, Untersuchungen zur Arbeitswelt der Frau in der römischen Antike, Frankfurt am Main, Peter Lang, 1988.

Evangelatou, Maria, "The Purple Thread of the Flesh: the Theological Connotations of a Narrative Iconographic Element in Byzantine Images of the Annunciation", in Icon and Word: the Power of Images in Byzantium. Studies presented to Robin Cormack, ed. by

A. Eastmond, L. James, Aldershot, Ashgate, 2003, p. 261-279.

Gury, Françoise, «La Forge du Destin. A propos d'une série de peintures pompéiennes du IV style», Mélanges de l'Ecole française de Rome. Antiquité, 98 (1986), p. 427-489.

Huskinson, Janet, Roman Children Sarcophagi. Their Decoration and its Social Significance, Oxford, Clarendon Press, 1996.

Jevtic, Ivana, "L'inscription dans la vie: la fileuse dans la nativité de la Vierge", Recueil des travaux de l'Institut d'études byzantines, 45 (2008), p. 169-176.

Kampen, Natalie Boymel, "Biographical Narration and Roman Funerary Art", American Journal of Archaeology, 85 (1981), p. $47-58$. 
Köves-Zulauf, Thomas, Römische Geburtsriten, München, C.H. Beck, 1990 (Zetemata Heft 87).

Matz, Friedrich, Die dionysischen Sarkophage, Berlin, Mann, 1969 (Die antiken Sarkophagreliefs, Bd IV, Teil 3).

Megow, Wolf-Ruidiger, "Priapos", LIMC VIII, Addendum (1994), p. 1028-1044.

Néraudau, Jean-Pierre, Etre enfant à Rome, Paris, Les Belles Lettres, 1984.

Olender, Maurice, "L'enfant Priape et son phallus", in Souffrance, plaisir et pensée, éd. par J. Caïn, A. de Mijolla, Paris, Les Belles Lettres, 1983, p. 141-164.

—, "Priape le mal taillé", in Corps des dieux, éd. par Ch. Malamoud, J.-P. Vernant, Paris, Gallimard, 1986, p. 373-388 (Le temps de la réflexion VII).

Rosenberger, Veit, Gezähmte Götter. Das Prodigienwesen der römischen Republik, Stuttgart, F. Steiner, 1998.

Samama, Evelyne, Les médecins dans le monde grec: sources épigraphiques sur la naissance d'un corps médical, Genève, Droz, 2003.

Santa Maria Scrinari, Vainea, Catalogo delle sculture romane. Museo archeologico di Aquileia, Roma, Istituto poligrafico dello Stato, 1972.

Schilling, R., "Recension de Léontine Louise Tels-de Jong: Sur quelques divinités romaines de la naissance et de la prophétie», Gnomon, 32 (1960), p. 650-653.

Schulz, Fritz, "Roman Registers of Birth and Birth Certificates", Journal of Roman Studies, 32 (1942), p. 78-91.

—, "Roman Registers of Birth and Birth Certificates. Part II», Journal of Roman Studies, 33 (1943), p. 55-64.

Shaw, Brent D., "Raising and Killing Children: Two Roman Myths", Mnemosyne, 54 (2001), p. 31-77.

Tels-de Jong, Léontine Louise, Sur quelques divinités romaines de la naissance et de la prophétie, Delft, Grafisch Bedrijf Avanti, 1959.

Thomas, Yan, "A Rome, père des citoyens et cité des pères (II ${ }^{e}$ siècle avant J.-C. - II siècle après J.-C.), in Histoire de la famille I, éd. par A. Burguière, Paris, A. Colin, 1986, p. 195-230.

WeIss, Carina, "Deae fata nascentibus canunt», in Kotinos. Festschrift für Erika Simon, Mainz, von Zabern, 1992, p. 366-374. 
West, Stephanie, «Whose Baby?: a Note on P. Oxy. 744 », Zeitschrift für Papyrologie und Epigraphik, 121 (1998), p. 167-172.

Yота, Elisabeth, "L'embryon dans l'art byzantin: une image insolite", in L'embryon humain à travers l'histoire. Images, savoirs et rites, éd. par V. Dasen, Gollion, Infolio, 2007, p. 83-106.

\section{Crédits photographiques}

Fig. 1, 4, 5, 7:

D'après Amedick, Rita, Vita Privata. Die Sarkophage mit Darstellungen aus dem Menschenleben, Berlin, Gebr. Mann, 1991 (Die antiken Sarkophagreliefs, Bd I, Teil 4), cat. no 64 , pl. 62,1; cat. no 49, pl. 62, 2; cat. $\mathrm{n}^{\mathrm{o}} 115$, pl. 56, 1 ; cat. $\mathrm{n}^{\mathrm{o}} 250$, pl. 62, 6 .

Fig. 2, 3, 8:

Photo DAI Rome neg. 8332, 33.I1, 82.439.

Fig. 6:

Dessin V. Dasen.

Fig. 9:

D’après Gury, Françoise, «La Forge du Destin. A propos d'une série de peintures pompéiennes du IVe style", Mélanges de l'Ecole française de Rome. Antiquité, 98 (1986), p. 462, fig. 14.

Fig. 10:

Photo du musée, Th. Blais-MAD.

Fig. 11:

(C) The Trustees of the British Museum.

Fig. 12:

D’après Furtwängler, Adolf, Die antiken Gemmen. Geschichte der Steinschneidekunst im klassischen Altertum, III, Leipzig/Berlin, Giesecke \& Devrient, 1900, p. 296, fig. 155. 
Fig. 13:

D’après Vocotopoulos, P., Greek Art: Byzantine Icons, Athen, Ekdotiki Athinon, 1955, fig. 49. 